\title{
Genetic profiling of two phenotypically distinct outbred rats derived from a colony of the Zucker fatty rats maintained at Tokyo Medical University
}

\author{
Satoshi NAKANISHI ${ }^{1)}$, Takashi KURAMOTO') ${ }^{1}$, Naomi KASHIWAZAKI'), and Norihide YOKOI ${ }^{3)}$ \\ 1) Institute of Laboratory Animals, Graduate School of Medicine, Kyoto University, Yoshidakonoe-cho, Sakyo-ku, \\ Kyoto 606-8501, Japan \\ ${ }^{2)}$ Graduate School of Veterinary Science, Azabu University, Sagamihara, Kanagawa 252-5201, Japan \\ ${ }^{3)}$ Division of Molecular and Metabolic Medicine, Department of Physiology and Cell Biology, Kobe University \\ Graduate School of Medicine, Kobe, Hyogo 650-0017, Japan
}

\begin{abstract}
The Zucker fatty (ZF) rat is an outbred rat and a well-known model of obesity without diabetes, harboring a missense mutation (fatty, abbreviated as $f a$ ) in the leptin receptor gene (Lepr). Slc:Zucker (SIc:ZF) outbred rats exhibit obesity while Hos:ZFDM-Lepra (Hos:ZFDM) outbred rats exhibit obesity and type 2 diabetes. Both outbred rats have been derived from an outbred ZF rat colony maintained at Tokyo Medical University. So far, genetic profiles of these outbred rats remain unknown. Here, we applied a simple genotyping method using Ampdirect reagents and FTA cards (Amp-FTA) in combination with simple sequence length polymorphisms (SSLP) markers to determine genetic profiles of SIc:ZF and Hos:ZFDM rats. Among 27 SSLP marker loci, 24 loci (89\%) were fixed for specific allele at each locus in Slc:ZF rats and 26 loci (96\%) were fixed in Hos:ZFDM rats, respectively. This indicates the low genetic heterogeneity in both colonies of outbred rats. Nine loci (33\%) showed different alleles between the two outbred rats, suggesting considerably different genetic profiles between the two outbred rats in spite of the same origin. Additional analysis using 72 SSLP markers further supported these results and clarified the profiles in detail. This study revealed that genetic profiles of the SIc:ZF and Hos:ZFDM outbred rats are different for about $30 \%$ of the SSLP marker loci, which is the underlying basis for the phenotypic difference between the two outbred rats.
\end{abstract}

Key words: Amp-FTA method, genetic profiling, SSLP markers, ZFDM rat, Zucker fatty rat

\section{Introduction}

The Zucker fatty (ZF) rat is a well-known model of obesity harboring a missense mutation (fatty, abbreviated as $f a$ ) in the leptin receptor gene (Lepr) on chromosome 5. The Leprfa (hereinafter referred to as " $f a$ ") mutation was originally discovered in crosses between Sherman and Merck stock M rats (13M strain) [18]. The $f a / f a$ homozygous rats are usually infertile and exhibit obesity, polyphagia, dyslipidemia and hyperinsulinemia, the phenotype of which is similar to human obesity. The infertility of $f a / f a$ homozygous animals requires maintenance and production of $f a /+$ heterozygous animals.

There are several outbred and inbred strains derived from the original ZF colony such as Crl:ZUC-Lepr ${ }^{f a}$ [1], Hos:ZFDM (Hos:ZFDM-Lepr ${ }^{f a}$ ) [14], Kwl:Zucker [5], Slc:ZF (Slc:Zucker) [14], or Zucker diabetic fatty (ZDF) rat [10]. Among them, the Slc:ZF and Hos:ZFDM rats have originated from an outbred ZF colony maintained at Tokyo Medical University. The Slc:ZF rats exhibiting

(Received 10 August 2016 / Accepted 11 October 2016 / Published online in J-STAGE 31 October 2016)

Address corresponding: S. Nakanishi, Institute of Laboratory Animals, Graduate School of Medicine, Kyoto University, Yoshidakonoe-cho, Sakyoku, Kyoto 606-8501, Japan

Supplementary Tables: refer to J-STAGE: https://www.jstage.jst.go.jp/browselexpanim

(C)2017 Japanese Association for Laboratory Animal Science 
obesity without diabetes have been established by Japan SLC, Inc. (Hamamatsu, Japan), while the Hos:ZFDM rats exhibiting obesity and type 2 diabetes have been established by Hoshino Laboratory Animals, Inc. (Ibaraki, Japan) [14]. It is noteworthy that $f a / f a$ homozygous male rats of the Hos:ZFDM colony are fertile. So far, genetic profiles of the Slc:ZF and Hos:ZFDM rats have not been reported. Genetic profiling of these two outbred rats allows for further insights into the genotype-based phenotypic difference between them.

The Amp-FTA method is a simple genotyping procedure which allows genomic DNA from unpurified blood immobilized on an FTA card to be amplified by polymerase chain reaction (PCR) when used with Ampdirect reagents $[6,8]$. Simple sequence length polymorphisms (SSLP) markers are based on 1-4 base pairs tandem repeats, which are characterized by high polymorphism, abundance, and random distribution throughout the genome. Since SSLP markers are easy to be analyzed by using PCR and gel electrophoresis, they have been utilized for construction of genetic maps [12], genetic analysis [3], and genetic monitoring of various rat strains [7]. In addition, SSLP markers are useful for clarifying genetic profiles of outbred rats [9].

The aim of the present work was to determine genetic profiles of Slc:ZF and Hos:ZFDM rats, both of which have been derived from an outbred ZF rat colony maintained at Tokyo Medical University. We first applied the Amp-FTA method in combination with 27 SSLP markers to determine genetic profiles of the two outbred rats. Secondly, we compared the genetic profiles of these rats with those of the KZ-Lepr ${ }^{f a} / \mathrm{Tky}$ (KZF) and KZC/Tky (KZC) inbred strains. These two rat strains have also been derived from the same ZF colony. Finally, we performed detailed genetic profiling of the Slc:ZF and Hos:ZFDM rats with additional 72 SSLP markers.

\section{Materials and Methods}

\section{Animals}

Female Hos:ZFDM rats $(f a / f a, \mathrm{n}=9 ; f a /+, \mathrm{n}=16 ;+++$, $\mathrm{n}=5$ ) were provided by Hoshino Laboratory Animals, Inc. (Ibaraki, Japan). Female Slc:ZF rats $(f a / f a, \mathrm{n}=8$; $f a /+, \mathrm{n}=18 ;+/+, \mathrm{n}=4$ ) were provided by Japan SLC, Inc. (Hamamatsu, Japan). All animal experiments were approved by the Committee on Animal Experimentation of Kobe University and carried out in accordance with the Guidelines for Animal Experimentation at Kobe University.

\section{Blood sampling with the FTA card}

Tip of tail (approximately $0.5-1.0 \mathrm{~mm}$ long) is cut from each animal under anesthesia by vaporing isoflurane. Several aliquots of blood are obtained from rubbed clipping tail and smeared on to the FTA card (GE Healthcare UK, Buckinghamshire, England). Blood samples on the FTA card were allowed to dry completely at room

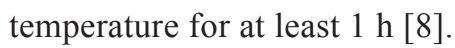

\section{Genomic DNA isolation}

Genomic DNA was extracted from the tail of Hos:ZFDM rats $(n=30)$ and Slc:ZF rats $(n=30)$ using a PI-200 automatic DNA extraction machine (KURABO INDUSTRIES Ltd., Osaka, Japan). Genomic DNA of the inbred KZF (KZ-Lepr ${ }^{f a} /$ Tky, NBRP Rat No.0032) and KZC (KZC/Tky, NBRP Rat No.0028) strains were provided by the National Bio Resource Project for the Rat (NBRP-Rat).

\section{Genotyping with the Amp-FTA method}

Twenty-seven SSLP markers were used for genotyping each rat to survey all chromosomes except for the $\mathrm{Y}$ chromosome (Fig. 1). PCR templates were prepared by punching discs out from blood-stained region on the FTA card which is $1.5 \mathrm{~mm}$ in diameter by using a conventional ear punch tool (NATSUME SEISAKUSHO Co., Ltd., Tokyo, Japan). Un-treated sample discs were placed directly in a $15 \mu 1$ PCR mixture containing the Ampdirect Plus reagent (SHIMADZU Corp., Kyoto, Japan), $0.2 \mu \mathrm{M}$ of each primer, and 0.4 units of BIO Taq Hot Start DNA polymerase (BIOLINE, UK). PCR was carried out under the following conditions: 94 degrees Celsius for $3 \mathrm{~min}$ and 40 cycles of 94 degrees Celsius for $30 \mathrm{~s}, 60$ degrees Celsius for $1 \mathrm{~min}$, and 72 degrees Celsius for $45 \mathrm{~s}$. PCR products were analyzed with the MultiNA Microchip Electrophoresis System (SHIMADZU Corp.). The allele types for each marker locus were determined based on the size of the PCR products and were called " $a$ ", " $b$ ", "c", and so on, in ascending order of size.

\section{Phylogenetic tree analysis}

The genotype data of the two outbred rats and two inbred strains on 27 SSLP markers were converted to allele format using the parallel Editor in GENETYX ver.10 (GENETYX Corp., Tokyo, Japan) (Supplemen- 

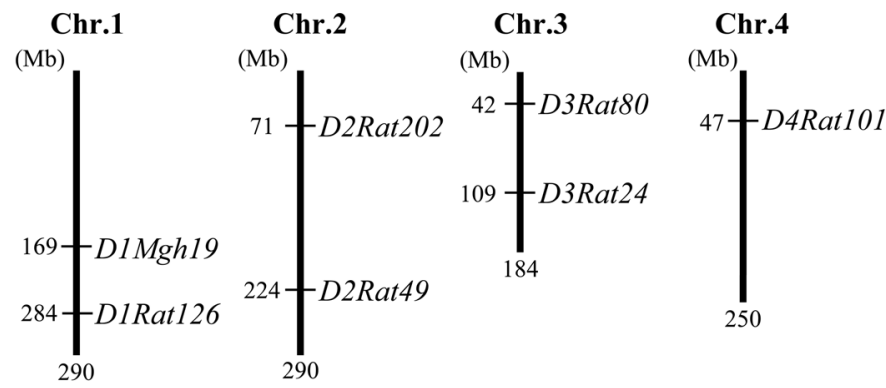
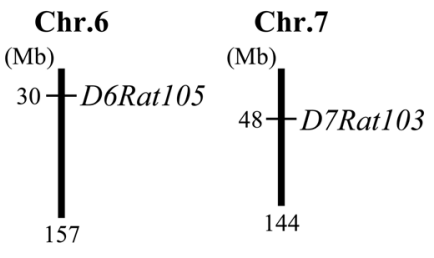
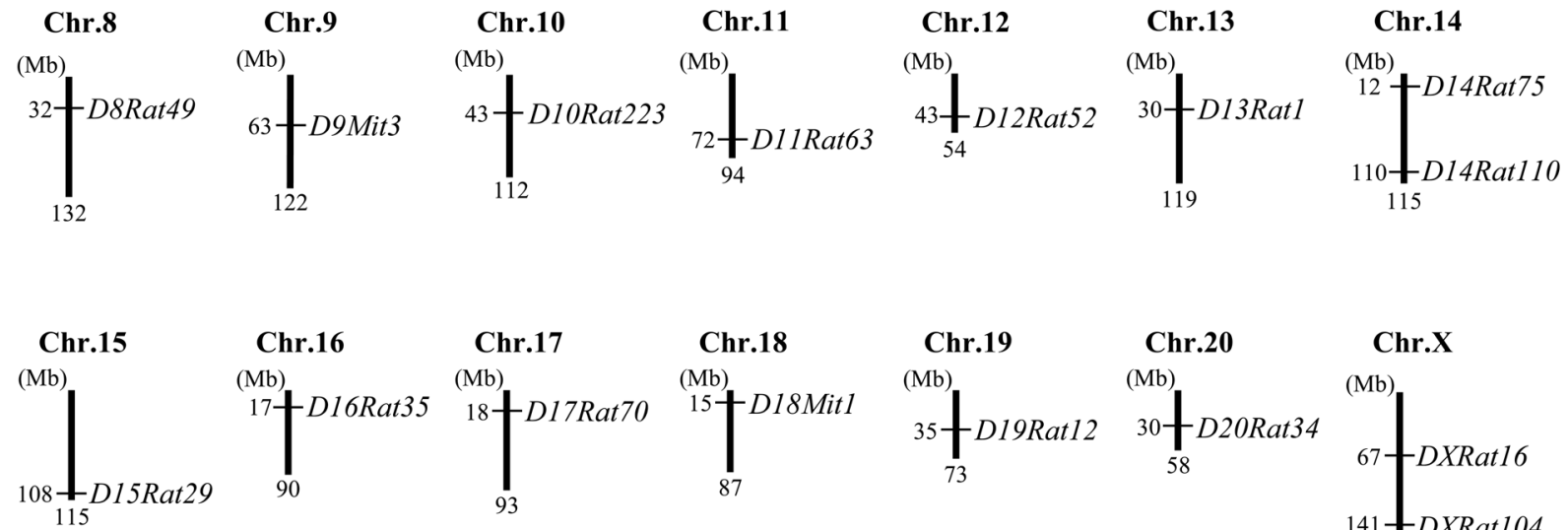

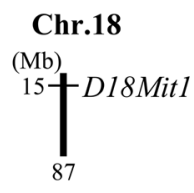

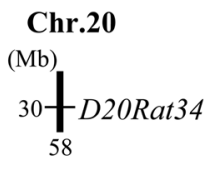

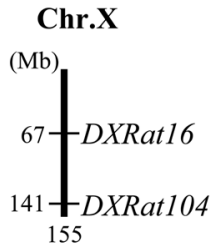

Fig. 1. Chromosomal location of the 27 SSLP makers in the rat genome. Twenty-seven markers are selected and used in combination with the Amp-FTA method to determine genetic profiles of the Hos:ZFDM and Slc:ZF outbred rats. Physical positions of markers and physical length of chromosomes (in $\mathrm{Mb}$ ) are based on the rat genome assembly: Rnor_5.0.

tary Table 1). An unrooted phylogenetic tree was obtained through UPGMA analysis implemented in GENETYX.

\section{Genetic profiling with 99 SSLP markers}

In addition to the 27 SSLP markers mentioned above, seventy-two SSLP markers were further used for genotyping of the Slc:ZF $(n=30)$ and Hos:ZFDM $(n=30)$ rats (Supplementary Table 2).

\section{Results}

Genetic profiling of the Slc:ZF and Hos:ZFDM outbred rats

To determine and compare genetic profiles of the Slc:ZF and Hos:ZFDM outbred rats, we first performed genotyping on 30 animals each using the Amp-FTA method in combination with 27 SSLP markers (Fig. 1). Specific and clear PCR products were obtained for all of the SSLP markers (Fig. 2). The alleles at each marker locus were called based on the size of the PCR prod- ucts and the allele frequencies were calculated from genotypes of 30 animals of each outbred rat. When only one allele type was observed at a particular locus, the locus was regarded as fixed. Among 27 SSLP marker loci, 24 loci (88\%) were fixed in the Slc:ZF colony and 26 loci (96\%) were also fixed in the Hos:ZFDM colony (Fig. 3A). Nine loci (33\%) showed different alleles between the two colonies of outbred rats. These results indicate that there is low genetic heterogeneity in both outbred rats and that these two outbred rats are genetically distinct to each other in spite of the same origin.

Phylogenetic tree analysis of the two outbred rats and two inbred strains derived from the same origin

To clarify the phylogenetic relationship of the two outbred rats and two inbred strains, all of which have been derived from the same ZF rat colony maintained at Tokyo Medical University, we determined genotypes of the 27 SSLP markers on the KZF and KZC inbred strains and compared those with the Slc:ZF and Hos:ZFDM rats (Fig. 3B). Alleles at all of the 24 fixed loci of the Slc:ZF colo- 
A

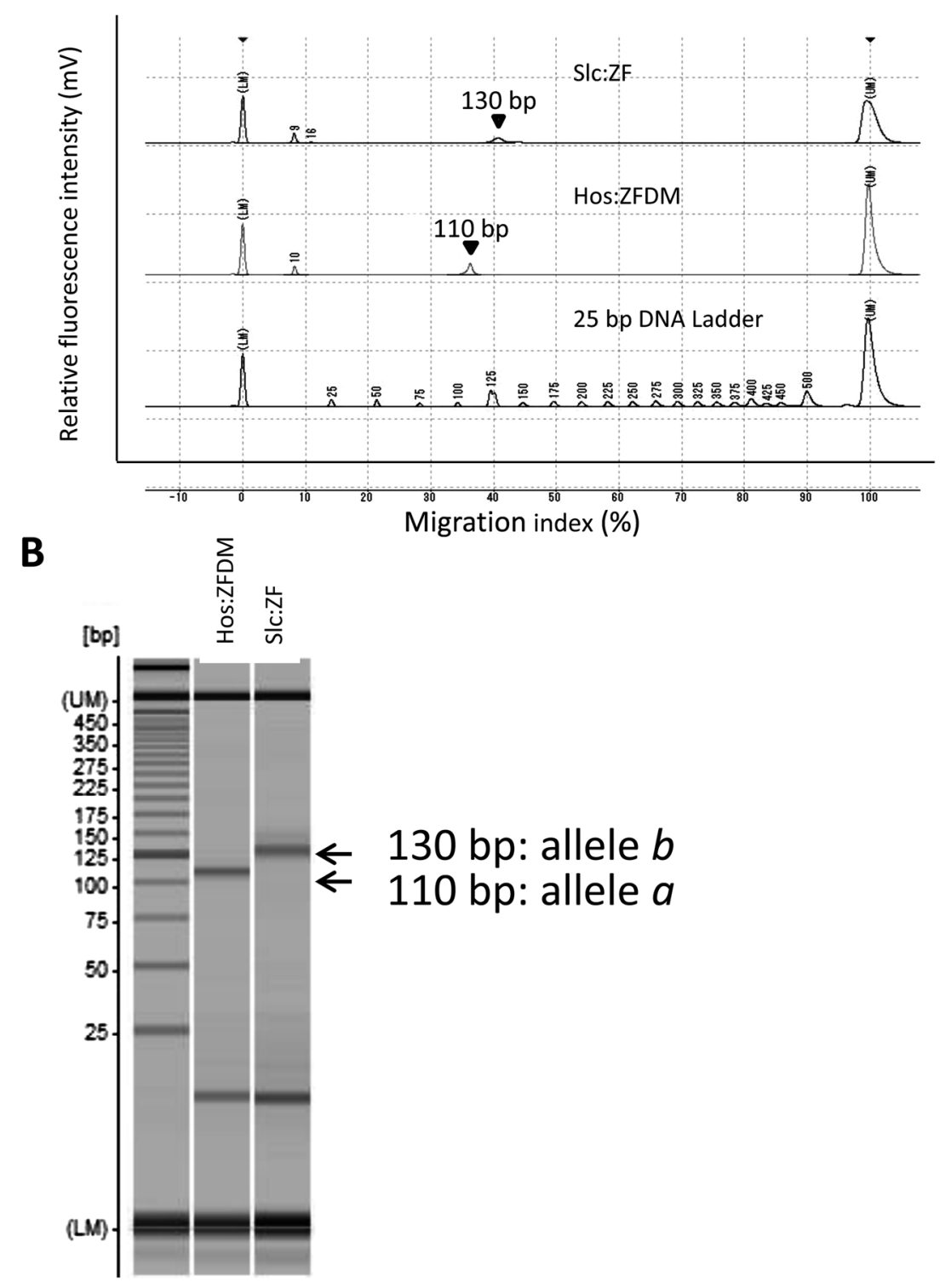

Fig. 2. Genotyping by using the MultiNA Microchip Electrophoresis System. A, Representative separation electropherogram image of the PCR products. Representative results of the SSLP marker (D20Rat34) are shown. Electrophoresis results were corrected by using 25 bp DNA ladder markers and two internal standard makers (LM, lower maker; UM, upper marker). B, Representative gel image of the PCR products. Representative results of the SSLP marker (D20Rat34) are shown. At this locus, two different PCR products were detected: the smaller (110 bp) and larger (130 bp) products were called "allele $a$ " and "allele $b$ ", respectively.

ny were identical to those of the KZF strain, indicative of the close phylogenetic relationship. The KZC strain had alleles different from the Slc:ZF at 7 loci (25\%) and those different from the Hos:ZFDM at 6 loci $(23 \%)$. The Hos:ZFDM showed alleles different from the KZF at 8 loci (30\%). Given the fact that 9 loci (33\%) showing different alleles between the Slc:ZF and Hos:ZFDM rats, phylogenetic distances between the Slc:ZF and KZC and between the Hos:ZFDM and KZC are similar to that between the Slc:ZF and Hos:ZFDM (Fig. 4). 
A

Hos:ZFDM

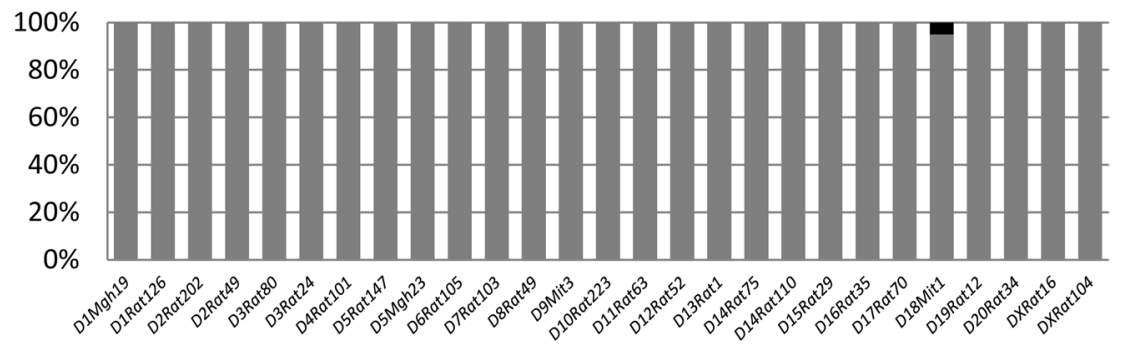

SIc:ZF

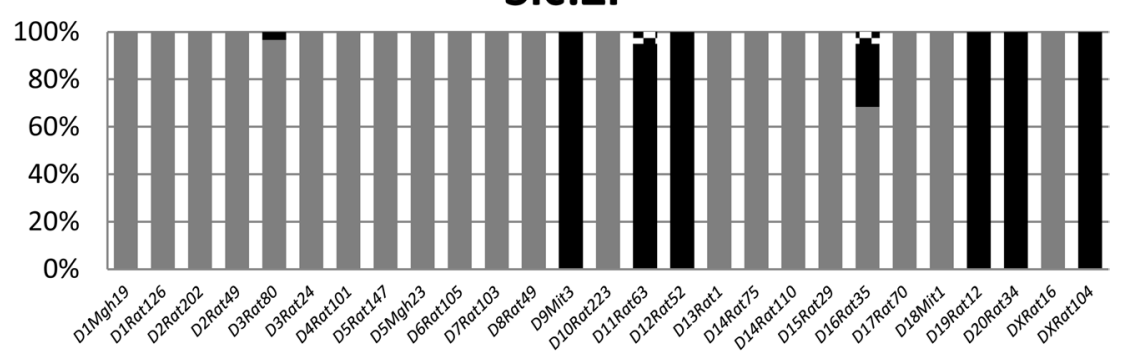

B

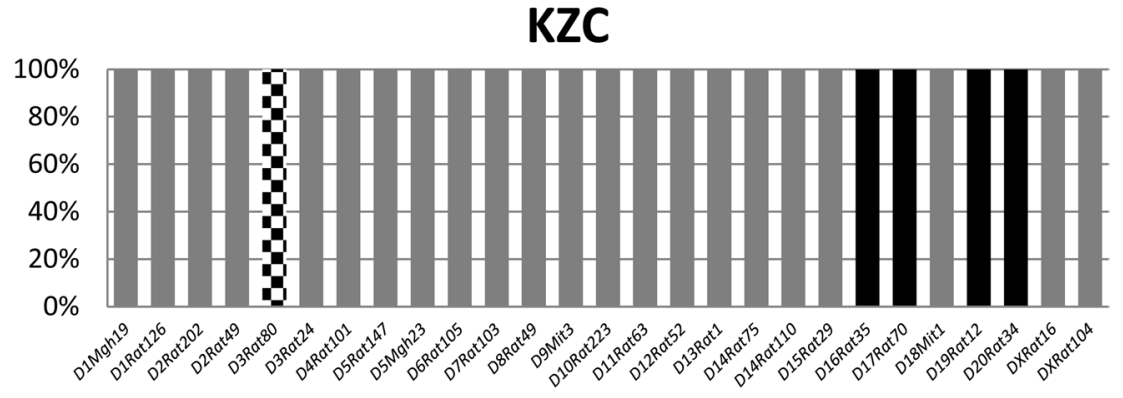

KZF

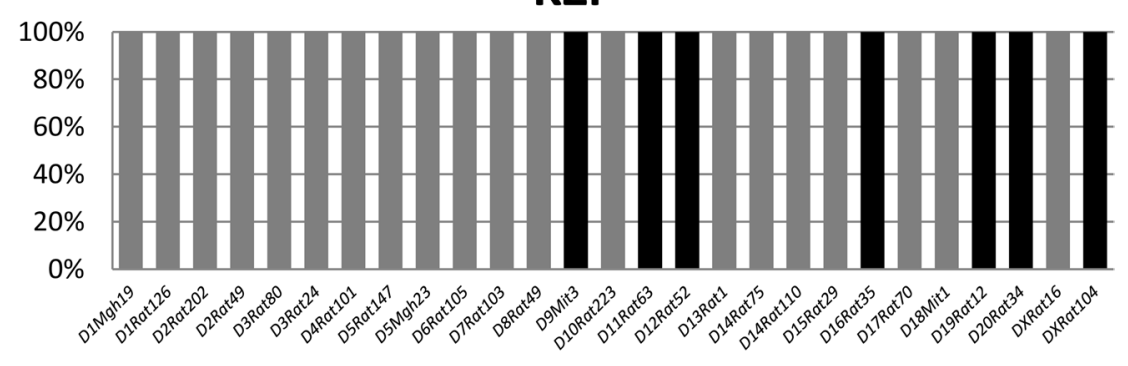

Fig. 3. Genetic profiles of the four strains derived from the same outbred ZF rat colony. A, Distribution of the 27 SSLP marker alleles in the Hos:ZFDM and Slc:ZF outbred rat colonies. Major alleles in the Hos:ZFDM colony are shown as solid grey bars. Other alleles are shown as black or diagonal bars. B, Distribution of the 27 SSLP marker alleles in the KZF and KZC inbred rat strains. Alleles that are identical to the major alleles in the Hos:ZFDM colony are shown as solid grey bars. Other alleles are shown as black or diagonal bars.

Detailed genetic profiling of the Slc:ZF and Hos:ZFDM outbred rats with 99 SSLP markers

To further clarify the deference in genetic profiles between the Slc:ZF and Hos:ZFDM outbred rats, we performed genotyping on the 30 animals each with additional 72 SSLP markers (Total of 99 SSLP markers). Among 99 SSLP marker loci, 93 loci (94\%) and 98 loci (99\%) were fixed in the Slc:ZF and Hos:ZFDM colonies, 
respectively (Fig. 5). In addition to the nine loci (33\%) showing different alleles between the two outbred rats among the 27 marker loci, 20 (28\%) out of 72 loci also showed different alleles between the two outbred rats

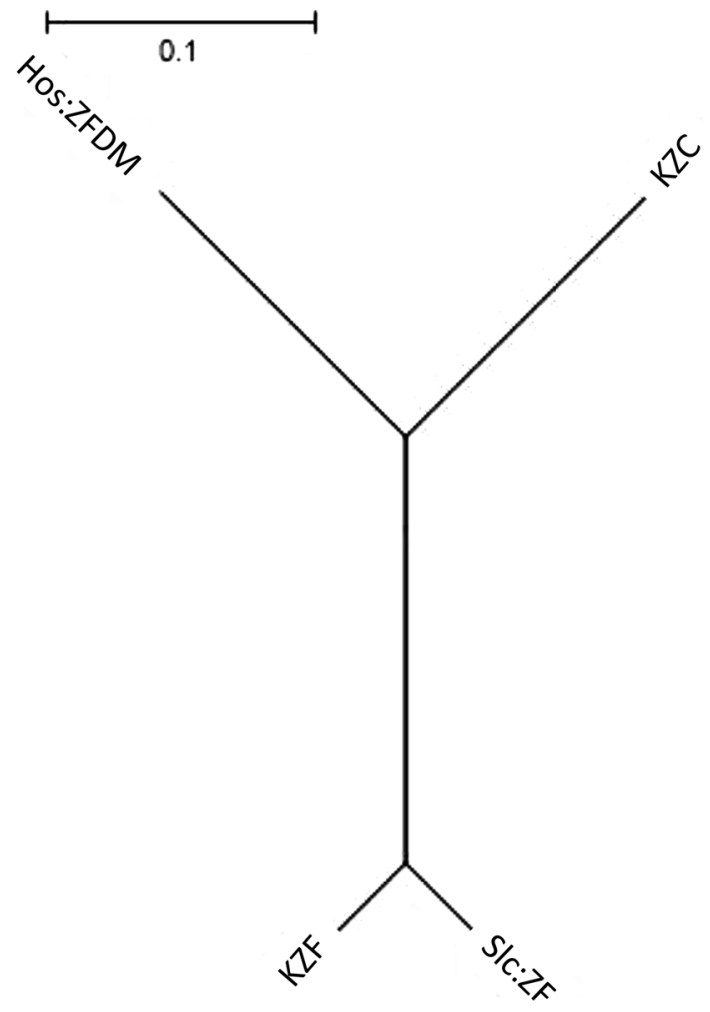

Fig. 4. Phylogenetic tree analysis of the four strains derived from the same outbred ZF rat colony. An unrooted phylogenetic tree for the four rat strains was obtained through UPGMA analysis implemented in the GENETYX software.
(In total, 29 out of 99 loci: 29\%). These results further support the findings of the relatively low genetic heterogeneity in both outbred rats and the considerably different genetic profiles of these outbred rats.

\section{Discussion}

In this study, we applied the Amp-FTA method in combination with SSLP markers to determine genetic profiles of the Slc:ZF and Hos:ZFDM outbred rats, both of which have been derived from the outbred ZF rat colony maintained at Tokyo Medical University. More than $90 \%$ of the marker loci were fixed in both outbred rats, indicating the relatively low genetic heterogeneity. About $30 \%$ of the marker loci showed different alleles between the two outbred rats, revealing the considerably different genetic profiles of these two outbred rats in spite of the same origin.

The Amp-FTA method has been developed as a simple and rapid genotyping method, which allows to perform PCR amplification of genomic DNA from blood without purification [8]. There is no need to use chemicals or equipment for purification of the genomic DNA, thus, there is no waste of time, resources and no pollution. In addition, the dried blood samples on the FTA cards can be stored at room temperature, and last at least 16 years [11]. By using this method in combination with SSLP markers, we here successfully determined genetic profiles of the two outbred rats. Accordingly, the combination of the Amp-FTA method with SSLP markers serves as a simple, rapid and useful method for genetic profiling.

\section{Hos:ZFDM}

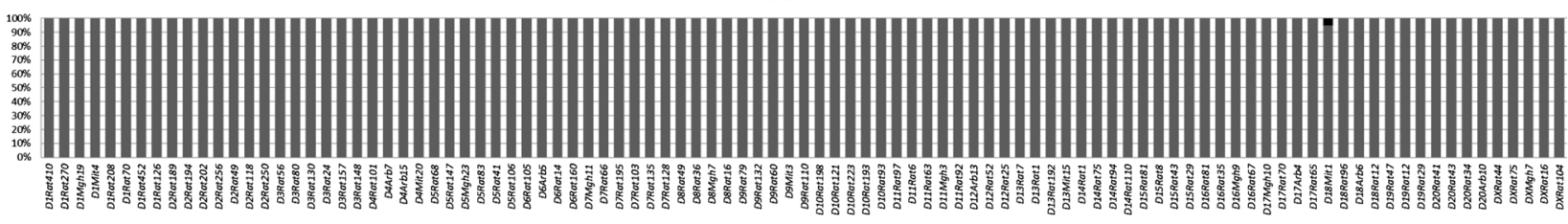

SIc:ZF

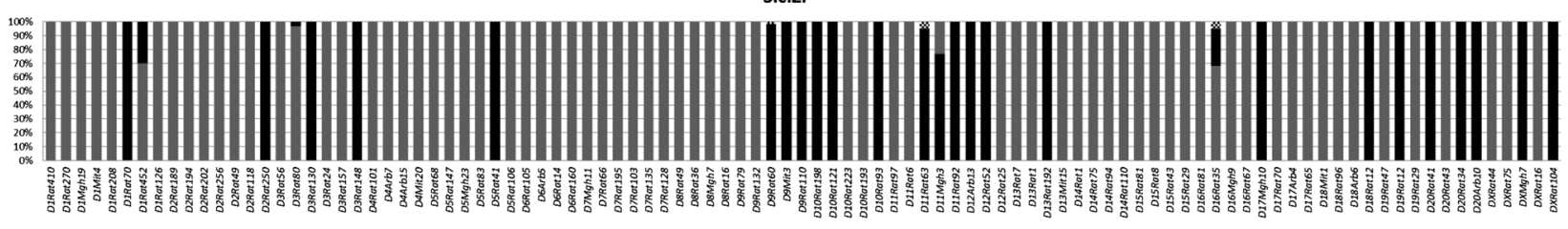

Fig. 5. Detailed genetic profiles of the Hos:ZFDM and Slc:ZF outbred rat colonies. Distribution of the 99 SSLP marker alleles in the Hos:ZFDM and Slc:ZF outbred rat colonies are shown. Major alleles in the Hos:ZFDM colony are shown as solid grey bars. Other alleles are shown as black or diagonal bars. 
Since the Slc:ZF and Hos:ZFDM outbred rats have been derived from the same ZF rat colony at Tokyo Medical University, genetic profiles of these outbred rats are thought to be similar. In addition, there are two inbred strains, KZF [17] and KZC [4], both of which have been derived from the same outbred ZF colony and have been established at Tokyo Medical University. The KZF rat is an inbred strain carrying the $f a$ mutation and exhibiting obesity [17], the phenotype of which is similar to that of the Slc:ZF rat. The KZC rat is an inbred strain carrying an autosomal recessive reelin (Reln) mutation causing severe ataxia and disarrangement of neuronal cells in the central nervous system, but without having the $f a$ mutation $[15,16]$. Although these four rats show distinct phenotypes, their genetic profiles are thought to be similar given the fact that these four rats have been derived from the same outbred ZF colony. However, the present study clearly showed that there is a considerable difference in their genetic profiles. Collectively, these findings suggest that there was a considerable genetic heterogeneity in the original ZF rat colony at Tokyo Medical University. In fact, different substrains have been derived from a colony of spontaneously hypertensive rat (SHR) at Kyoto University and genetic differences among them vary from 6 to $29 \%$ [7].

Differences in genetic profiles between the Slc:ZF and Hos:ZFDM rats may underlie the differences in their phenotypes. The Slc:ZF rats show obesity only, whereas the Hos:ZFDM rats exhibit obesity and type 2 diabetes [14]. The falfa homozygous males of the Hos:ZFDM rats have reproductive ability, while those of the Slc:ZF rats do not [14]. During the establishment of the Hos:ZFDM rats, selective breeding has been applied to maintain reproductive ability and higher blood glucose levels [14], leading to accumulation and nearly fixation of alleles responsible for reproductive ability and type 2 diabetes. The different genomic regions between the two outbred rats would be potential candidate regions involved in the development of type 2 diabetes and their reproductive ability. Indeed, among the 29 loci which showed different alleles between the two outbred rats, 16 loci harbored quantitative trait loci (QTLs) that were associated with diabetes and related traits (Supplementary Table 3) [13]. These QTLs might be involved in the development of type 2 diabetes in the Hos:ZFDM rats. Comparison of genetic profiles of the two outbred rats with other available ZF colony including the ZDF strain could be useful for clarifying genomic regions involved in the develop- ment of type 2 diabetes and reproductive ability. The low genetic heterogeneity in the two outbred rats guarantees the low variation in their phenotypes. In fact, diabetes incidence in $f a / f a$ homozygous male Hos:ZFDM rats reaches $100 \%[2,14]$. Accordingly, genetic analysis of the cross between the Slc:ZF and Hos:ZFDM rats would help us to identify genes responsible for reproductive ability and type 2 diabetes.

In conclusion, genetic profiles of the Slc:ZF and Hos:ZFDM outbred rats are revealed to be different for about $30 \%$ of the SSLP marker loci in spite of the same origin, which may explain the phenotypic difference between the two outbred rats. The genetic profiles obtained in the present study would contribute to the elucidation of genetic factors involved in the development of type 2 diabetes.

\section{Conflict of Interest}

The authors have no conflict of interest to declare.

\section{Acknowledgments}

We thank Japan SLC, Inc. and Hoshino Laboratory Animals, Inc. for providing Slc:Zucker and Hos:ZFDM rats, respectively. We also thank NBRP-Rat for providing genomic DNA of the KZF and KZC rats. This study was supported by Grants-in-Aid for Scientific Research from the Ministry of Education, Culture, Sports, Science and Technology, Japan. Part of the study was also supported by research grants from the NBRP-Rat and the Kobayashi Foundation.

\section{References}

1. Frisbee, J.C. 2005. Hypertension-independent microvascular rarefaction in the obese Zucker rat model of the metabolic syndrome. Microcirculation 12: 383-392. [Medline] [CrossRef]

2. Gheni, G., Yokoi, N., Beppu, M., Yamaguchi, T., Hidaka, S., Kawabata, A., Hoshino, Y., Hoshino, M., and Seino, S. 2015. Characterization of the prediabetic state in a novel rat model of type 2 diabetes, the ZFDM Rat. J. Diabetes Res. 2015: 261418. [Medline] [CrossRef]

3. Hilbert, P., Lindpaintner, K., Beckmann, J.S., Serikawa, T., Soubrier, F., Dubay, C., Cartwright, P., De Gouyon, B., Julier, C., Takahasi, S., Vincent, M., Ganten, D., Georges, M., and Lathrop, G.M. 1991. Chromosomal mapping of two genetic loci associated with blood-pressure regulation in hereditary hypertensive rats. Nature 353: 521-529. [Medline] [CrossRef] 
4. Ishibashi, K., Komeda, K., Sekiguchi, F., and Kanazawa, Y. 1989. Creeping: a new mutant rat with neurological disease. Lab. Anim. Sci. 39: 132-136. [Medline]

5. Isozaki, O., Tsushima, T., Miyakawa, M., Nozoe, Y., Demura, H., and Seki, H. 1999. Growth hormone directly inhibits leptin gene expression in visceral fat tissue in fatty Zucker rats. J. Endocrinol. 161: 511-516. [Medline] [CrossRef]

6. Kuramoto, T., Nakanishi, S., Yamasaki, K., Kumafuji, K., Sakakibara, Y., Neoda, Y., Takizawa, A., Kaneko, T., Otsuki, M., Hashimoto, R., Voigt, B., Mashimo, T., and Serikawa, T. 2010. Genetic quality control of the rat strains at the National Bio Resource Project - Rat. IBC 2: 1-8.

7. Mashimo, T., Voigt, B., Tsurumi, T., Naoi, K., Nakanishi, S., Yamasaki, K., Kuramoto, T., and Serikawa, T. 2006. A set of highly informative rat simple sequence length polymorphism (SSLP) markers and genetically defined rat strains. BMC Genet. 7: 19. [Medline] [CrossRef]

8. Nakanishi, S., Kuramoto, T., and Serikawa, T. 2009. Simple genotyping method using Ampdirect ${ }^{\circledR}$ Plus and FTA ${ }^{\circledR}$ technologies: application to the identification of transgenic animals and their routine genetic monitoring. Lab. Anim. Res. 25: 75-78.

9. Nakanishi, S., Serikawa, T., and Kuramoto, T. 2015. Slc:Wistar outbred rats show close genetic similarity with F344 inbred rats. Exp. Anim. 64: 25-29. [Medline] [CrossRef]

10. Peterson, R.G., Shaw, M.W., Neel, M.A., Little, L.A., and Eichberg, J. 1990. Zucker diabetic fatty rat as a model for non-insulin dependent diabetes mellitus. ILAR J. 32: 16-19. [CrossRef]

11. Rahikainen, A.L., Palo, J.U., de Leeuw, W., Budowle, B., and Sajantila, A. 2016. DNA quality and quantity from up to 16 years old post-mortem blood stored on FTA cards. Foren- sic Sci. Int. 261: 148-153. [Medline] [CrossRef]

12. Serikawa, T., Kuramoto, T., Hilbert, P., Mori, M., Yamada, J., Dubay, C.J., Lindpainter, K., Ganten, D., Guénet, J.L., Lathrop, G.M., and Beckman, J.S. 1992. Rat gene mapping using PCR-analyzed microsatellites. Genetics 131: 701-721. [Medline]

13. Shimoyama, M., De Pons, J., Hayman, G.T., Laulederkind, S.J., Liu, W., Nigam, R., Petri, V., Smith, J.R., Tutaj, M., Wang, S.J., Worthey, E., Dwinell, M., and Jacob, H. 2015. The Rat Genome Database 2015: genomic, phenotypic and environmental variations and disease. Nucleic Acids Res. 43: D743-D750. [Medline] [CrossRef]

14. Yokoi, N., Hoshino, M., Hidaka, S., Yoshida, E., Beppu, M., Hoshikawa, R., Sudo, K., Kawada, A., Takagi, S., and Seino, S. 2013. A novel rat model of type 2 diabetes: the Zucker fatty diabetes mellitus ZFDM rat. J. Diabetes Res. 2013: 103731. [Medline] [CrossRef]

15. Yokoi, N., Namae, M., Wang, H.Y., Kojima, K., Fuse, M., Yasuda, K., Serikawa, T., Seino, S., and Komeda, K. 2003. Rat neurological disease creeping is caused by a mutation in the reelin gene. Brain Res. Mol. Brain Res. 112: 1-7. [Medline] [CrossRef]

16. Yokoi, N., Shimizu, S., Ishibashi, K., Kitada, K., Iwama, H., Namae, M., Sugawara, M., Serikawa, T., and Komeda, K. 2000. Genetic mapping of the rat mutation creeping and evaluation of its positional candidate gene reelin. Mamm. Genome 11: 111-114. [Medline] [CrossRef]

17. Zhao, L.C., Zhang, X.D., Liao, S.X., Gao, H.C., Wang, H.Y., and Lin, D.H. 2010. A metabonomic comparison of urinary changes in Zucker and GK rats. J. Biomed. Biotechnol. 2010: 431894. [Medline] [CrossRef]

18. Zucker, L.M. and Zucker, T.F. 1961. Fatty, a new mutation in the rat. J. Hered. 52: 275-278. 